\section{A Ball-mill Emulsifier for 3-5 ml. Volumes}

\section{G. I. C. INGRAM}

From the Louis Jenner Laboratory and Department of Clinical Pathology, St. Thomas's Hospital and Medical School, London

\section{(RECEIVED FOR PUBLICATION DECEMBER 11, 1958)}

The present interest shown by workers on problems of coagulation in intractable, waxy phospholipids has prompted the development of an emulsifier to handle small volumes. The device has also been used for preparing even suspensions of inorganic precipitates and of platelet material.

\section{Construction}

Principle.-A polythene paddle revolved rapidly by a toy electric motor agitates the liquid in a robust vessel and throws into violent motion a number of glass beads which grind and pound as in a ball-mill.

Method.-The assembly is shown in Figs. 1 and 2. A convenient robust vessel is the lower portion of a Chalk's bottle (which has a rounded cavity, without a corner) held by the neck in a clamp attached to the leg of a tripod. Upon this is mounted the motor, e.g., a $6 \mathrm{v}$. " mighty midget" without reduction gear, or a $6 \mathrm{v}$. or $12 \mathrm{v}$. " micro motor mark III" which may be run at low temperatures (Victory Industries (Surrey) Ltd., Guildford). The tripod also supports the batteries, with as much switching gear as may be thought worth while.

Fig. 3 indicates the relations of the main components. The construction of the paddle and its coupling from Nos. 3 and 4 polythene catheter and copper wire is illustrated in Fig. 4. A somewhat elaborate but convenient electrical circuit is given in Fig. 5 .

Convenience is further served by partially closing the neck of the bottle around the shaft of the paddle by fitting a suitably punched polythene bottle closure to prevent splashing, and also by attaching a suction lead, as indicated, to increase the evaporation of organic solvents.
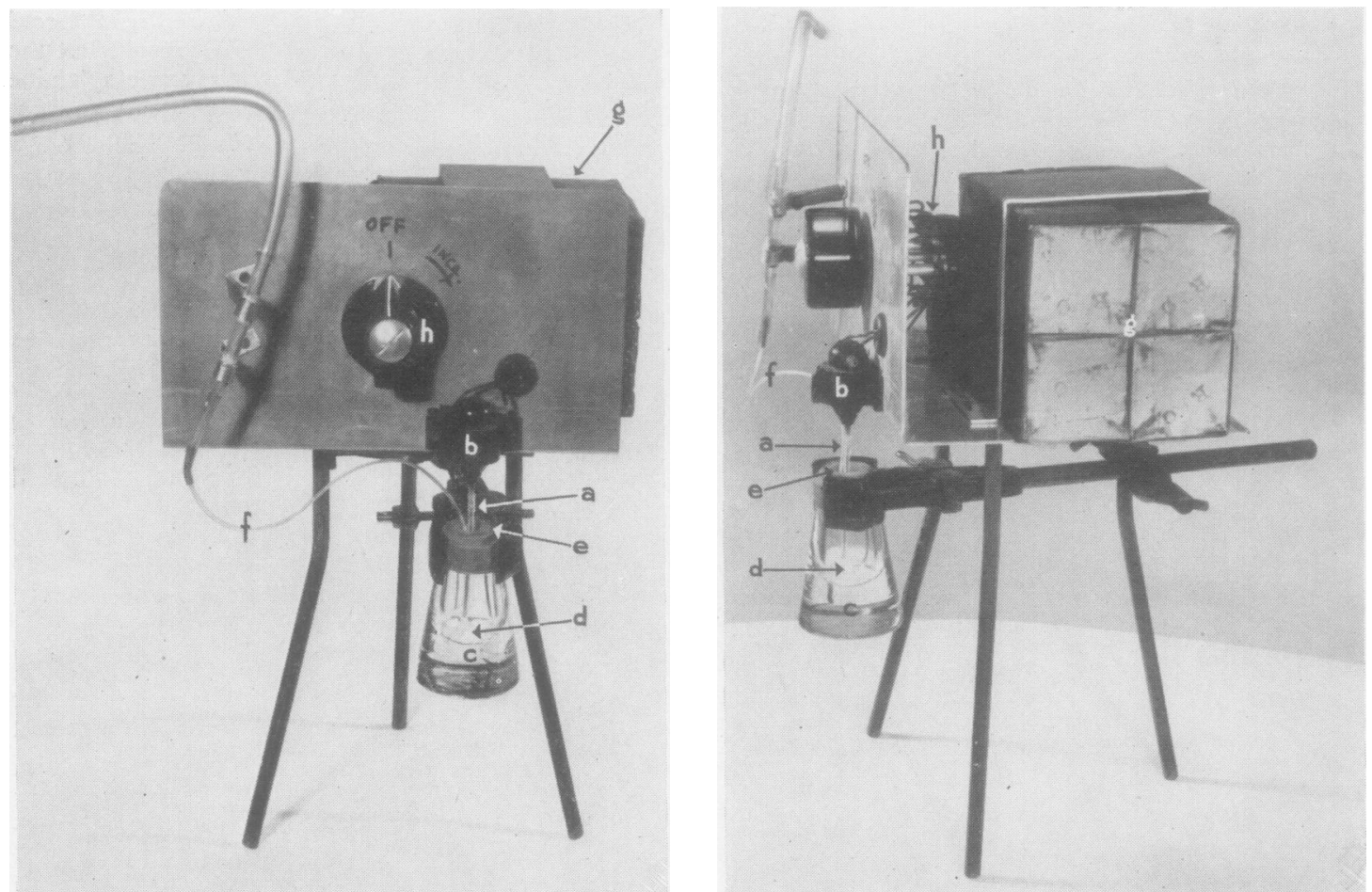

Figs. 1 and 2.-Front and side views showing the assembled emulsifier. $a=$ Paddle. $b=$ Motor. $c=$ Chalk's bottle, containing glass beads (the appearance of being half-full of liquid is given by the very thick base of the bottle). $\mathrm{d}=\mathrm{Glass}$ beads. $\mathrm{e}=\mathrm{Plastic}$ bottle-closure, separately punched for the paddle to pass loosely and for the tip of the suction line to fit tightly. $f=$ Suction line. $\mathrm{g}=$ Four bell-batteries. $\mathrm{h}=$ Incremental resistance. 


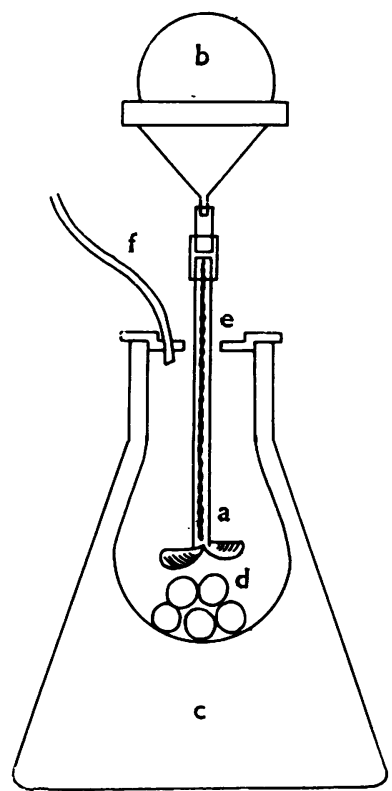

FIG. 3.-Diagram of the relations of the main components. (The key le.ters are the same as in Figs. 1 and 2.)

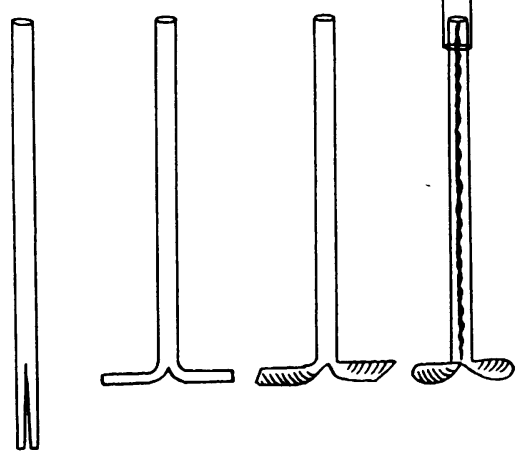

Fig. 4.-Construction of the paddle. A length of No. 3 polythene catheter is split at one end and the flaps separated after immersion in boiling water. The blades of the paddle are moulded with hot forceps and trimmed to the desired size. The shaft is stiffened with wire. The lower end may be sealed with dental cement. The upper end mounts a coupling of No. 4 catheter.

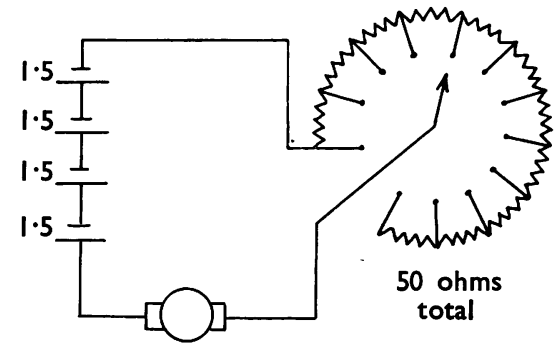

FIG. 5.-A suggested wiring diagram. On the original model, the coil of the incremental speed control resistance was wound by hand, giving 10 positions for the selector switch. A simpler arrangement would be to have four positions representing respectively one, two, three, and four batteries in series.

\section{Use}

To prepare a phospholipid " emulsion," pipette the required volume of aqueous vehicle into the Chalk's bottle, e.g., $2 \mathrm{ml}$. saline. Weigh out the required quantity of phospholipid in a $5 \mathrm{ml}$. beaker, e.g., $6 \mathrm{mg}$. Dissolve in ether, say $1 \mathrm{ml}$. and transfer, with ethereal washings, to the Chalk's bottle. Assemble the emulsifier, apply suction, and start the motor slowly. The ether is evaporated, leaving the phospholipid as a curd in the bottle. Detach suction and increase the speed of the motor until the contents of the bottle are being vigorously churned. Continue until emulsion forms (5-10 $\mathrm{min}$.).

It is a pleasure to acknowledge my indebtedness to a number of colleagues, especially to Dr. D. C. Simpson and Mr. J. Leiper, lately of the Department of Surgery, University of Edinburgh, who respectively suggested the motor and the polythene paddle ; to Mr. P. R. Styles and the staff of the Medical Electronics Department of St. Thomas's Hospital, who developed the electric circuit; to Miss P. Leicester, of St. Thomas's Hospital Medical School, for the diagrams ; and to the Photographic Department of St. Thomas's Hospital. 